\title{
Time for new imaging and therapeutic approaches in cardiac amyloidosis
}

\author{
Riemer H. J. A. Slart ${ }^{1,2,3} \cdot$ Andor W. J. M. Glaudemans ${ }^{1,3} \cdot$ Walter Noordzij $^{1,3} \cdot$ Johan Bijzet $^{3,4}$. \\ Bouke P. C. Hazenberg ${ }^{3,4}$ • Hans L. A. Nienhuis ${ }^{3,5}$
}

Received: 20 March 2019 / Accepted: 31 March 2019 / Published online: 23 April 2019

(C) Springer-Verlag GmbH Germany, part of Springer Nature 2019

Cardiac amyloidosis (CA), commonly resulting from deposition of misfolded immunoglobulin light chain (AL) or transthyretin (ATTR) protein, is an underestimated cause of heart failure [1, 2]. ATTR has gained increasing attention in recent years and can be divided into a hereditary type (ATTRv) and a wild-type (ATTRwt) [3]. Diagnosis of CA is frequently delayed for several reasons [4]. Clinical manifestations are varied, serum cardiac biomarker elevation is non-specific, awareness of CA is lacking, and noninvasive techniques for specific diagnosis became only more recently available. In patients with heart failure with preserved ejection fraction (HFpEF), moderate or severe interstitial amyloid deposition is present in 5-13\% of the cases, while mild interstitial and/or intramural coronary vascular deposition was present in $12 \%[2,5]$.

Selective treatment is delayed in a substantial proportion of the affected individuals because of this late recognition. Accurate and early diagnosis of heart failure as a result of CA has major implications on prognosis and treatment. CMR imaging with late gadolinium enhancement and T1

This Editorial Commentary refers to the article https://doi.org/10.1007/ s00259-019-04290-y

Riemer H. J. A. Slart

r.h.j.a.slart@umcg.nl

1 Medical Imaging Center, Department of Nuclear Medicine and Molecular Imaging, University of Groningen, University Medical Center Groningen, Groningen, The Netherlands

2 Department of Biomedical Photonic Imaging, TechMed Centre, University of Twente, Enschede, The Netherlands

3 Amyloidosis Center of Expertise, University of Groningen, University Medical Center Groningen, Groningen, The Netherlands

4 Department of Rheumatology \& Clinical Immunology, University of Groningen, University Medical Center Groningen, Groningen, The Netherlands

5 Department of Internal Medicine, University of Groningen, University Medical Center Groningen, Groningen, The Netherlands mapping may be helpful but is not able to reliably differentiate between cardiac amyloidosis due to ATTR or to other types of amyloidosis. However, assessment of extracellular volume using quantitative T1 mapping (either native or before and after application of contrast agent) has been reported as a promising biomarker for moderate micro-morphological changes, which presumably are present in early stages of the disease [6]. Molecular imaging with PET and SPECT nowadays play a critical role in the diagnosis, identification and distinction between ATTR and AL type CA.

Figure 1 shows the pathogenesis of amyloid deposition for the three major types, i.e. AL, ATTR and AA type in the heart. Increased thickness of the ventricular walls and strain differences (apical sparing) caused by extracellular deposition of amyloid can be detected by echocardiography. Delayed enhancement of gadolinium using MRI reflects an increased extravascular volume of the myocardial tissue. Serum amyloid $\mathrm{P}$ component (SAP) binds in a calcium-dependent way to all types of amyloid in many vital organs, but when labeled with ${ }^{123}$ I, not to amyloid in the heart [7]. This may be caused by the lack of a fenestrated endothelium in the myocardium, hindering access of the large $125 \mathrm{kDa}$ tracer to the extracellular space. Florbetapen is closely related to thioflavin T, a strong stain of amyloid that binds to repetitive motifs at the surface of the fibrils [8]. Bisphosphonates and pyrophosphate bind strongly to ATTR amyloid and weakly or not at all to AA and $\mathrm{AL}$ amyloid. Although there may be a relation with microcalcifications [9], the bone tracer ${ }^{18} \mathrm{~F}-\mathrm{NaF}$ does not always show the same strong tracer retention in ATTR CA [10]. The specific binding is probably not only to calcium, but technetium can bind to some metals [11] and/or to sulfhydryl groups on ATTR amyloid [12, 13]. Aprotinin has been used in the past to detect CA [14] and it may bind to repetitive motifs and/or electrostatically [15]. MIBG normally accumulates in vesicles in sympathetic nerve endings close to myocardial cells and the reduced uptake and increased loss probably reflects myocardial cell damage caused by amyloid [16]. 
AA

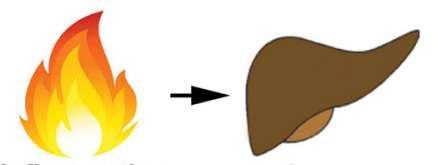

inflammation

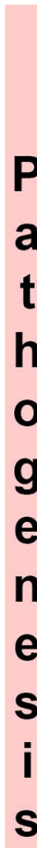

P
a
t
h
o
g
e
h
e
s
i
s
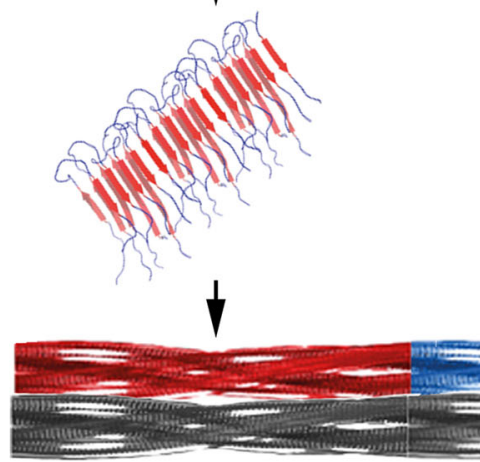

P
a
h
o
$g$
e
n
e
s
i
s

SAA

$\downarrow$

P
a
t
h
o
g
e
n
e
s
i
s

P
a
t
h
o
g
e
h
e
s
i
s

P
a
t
h
o
g
e
h
e
s
i
s

P
a
t
h
o
g
e
h
e
s
i
s
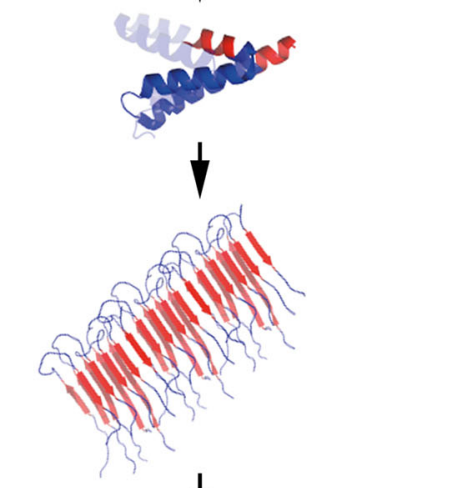

AL
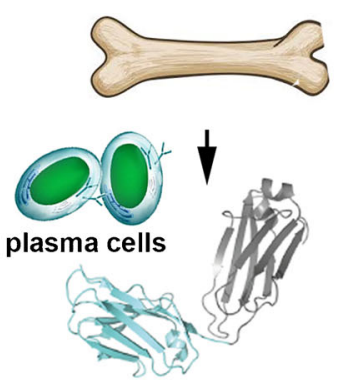

light chain

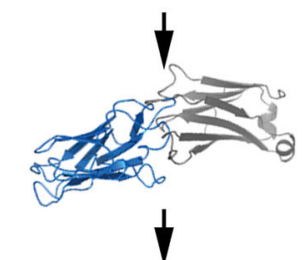

ATTR

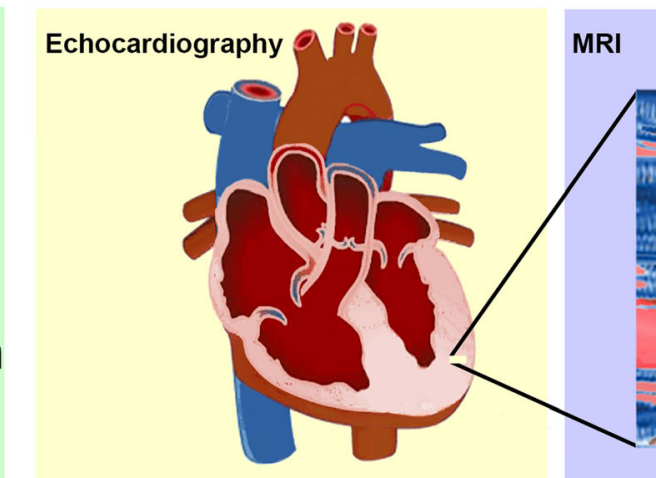

g

i

n

g

Molecular Imaging
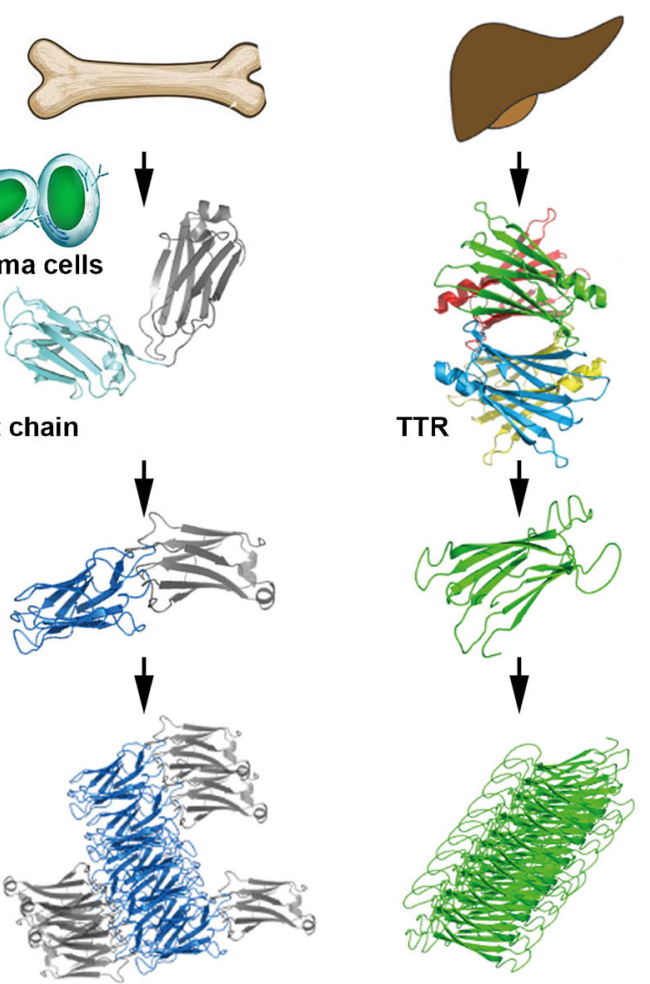

$\downarrow$

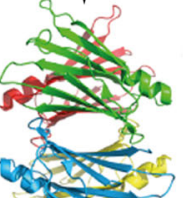

secretion of

precursor

proteins

TTR

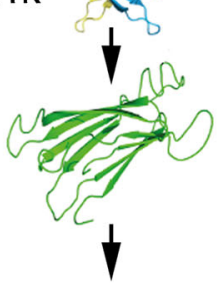

dissociation

and

misfolding

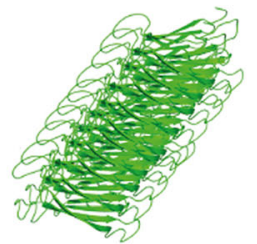

aggregation

amyloid fibril

formation and

deposition

e.g in the heart

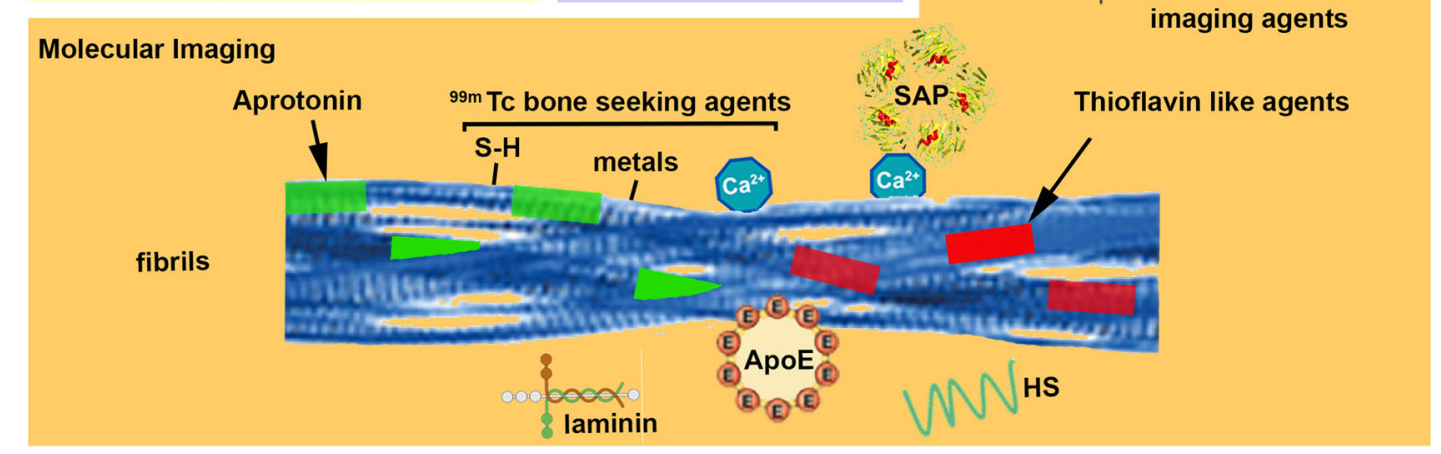


4 Fig. 1 In the upper part the pathogenic pathways of AA, AL and ATTR, the major three types of amyloidosis, are shown vertically. In AA, chronic inflammation stimulates the liver to produce the acute phase protein SAA, that becomes cleaved and misfolded. In AL, clonal plasma cells in the bone marrow produce immunoglobulin light chains, of which the variable part becomes misfolded. In ATTR, the liver produces the tetrameric protein transthyretin, that dissociates into monomers that become misfolded. The misfolded precursor proteins aggregate, finally resulting in the formation of amyloid fibrils (AA shown in red, $\mathrm{AL}$ in blue, and ATTR in green). Without specific immunohistochemical staining, fibrils of different types are indistinguishable from each other under the microscope (shown in grey)

The lower part of the figure is focused on imaging of amyloid deposition in the extracellular myocardial tissue. Echocardiography reveals increased wall thickness and stiffness, whereas late gadolinium enhancement in MRI reflects an increased volume of the extracellular myocardial tissue. Mechanisms used for molecular imaging of amyloid show specific binding of tracers to amyloid-specific elements of fibrils and related extracellular matrix in which the amyloid is anchored. Connected to amyloid in the extracellular matrix an increase of molecules such as serum amyloid P component (SAP), heparan sulphate (HS), apolipoprotein E (apoE), and laminin is found. Aprotinin and thioflavin-like agents ( $\mathrm{PiB}$, florbetapir and florbetapen) bind directly to repetitive motifs on the exterior surface of the fibrils. Technetium-labelled bone seeking agents (pyrophosphate and bisphosphonates) may bind to calcium, but also to metals ( $\mathrm{Zn}, \mathrm{Fe}, \mathrm{Cu}, \mathrm{Mn}$ and $\mathrm{Ba}$ ) and sulfhydryl ( $\mathrm{S}-\mathrm{H})$ groups on the ATTR amyloid fibril. Cardiac innervation imaging agents (e.g. ${ }^{123} \mathrm{I}-$ MIBG, ${ }^{11} \mathrm{C}$-mHED) accumulate less than expected in the nerve endings, as reflected in reduced cardiac tracer uptake and enhanced wash-out

In the current study of Kircher and colleagues in this issue, the performance of ${ }^{18} \mathrm{~F}$-florbetaben-PET/CT in the detection of CA (AL, ATTR and AA) was examined in 22 patients (5 histologically proven and 17 clinically suspected) and compared to echocardiography, CMR and ${ }^{99 \mathrm{~m}}$ Tc-DPD bone scintigraphy [17]. Additionally, the use of ${ }^{18} \mathrm{~F}$-florbetaben-PET/ CT for quantification of amyloid burden, including myocardial tracer retention (MTR), and monitoring of treatment response was assessed. Myocardial ${ }^{18} \mathrm{~F}$-florbetaben retention was found consistent with CA in 14/22 patients. Suspicion of CA was subsequently dropped in all eight PET-negative patients. Amyloid subtypes showed characteristic retention patterns (AL > AA > ATTR; all $p<0.005)$. MTR correlated with morphologic and functional parameters, as measured by CMR and echo (all $r>0.47$, all $p<0.05$ ), but not with cardiac biomarkers. Changes in MTR from baseline to follow-up corresponded well to treatment response, as assessed by cardiac biomarkers and performance status.

The authors concluded that imaging of CA with ${ }^{18} \mathrm{~F}$ florbetaben PET/CT is feasible and might be useful in differentiating CA subtypes. Although this study consisted of a small, heterogeneous patient cohort, including treatmentnaïve as well as pre-treated patients, it underlines the clinical value of applying new more specific PET imaging tracers in cardiac imaging. To demonstrate, however, the potential of amyloid-directed PET as a non-invasive instrument of (early) therapy monitoring, an understanding of the underlying biology is of utmost importance. Histological proof was lacking in the present study, including additional whole-body imaging to detect further sites of organ involvement in systemic amyloidosis, and this should be addressed in future studies.

More specific PET-imaging tracers in amyloidosis selectively bind to $\beta$-amyloid plaques and were originally designed as an aid to establish the clinical diagnosis of Alzheimer's disease. These specific radiopharmaceuticals are the benzothiazoles ${ }^{11} \mathrm{C}$-Pittsburgh compound-B $\left({ }^{11} \mathrm{C}-\mathrm{PiB}\right)$ and ${ }^{18} \mathrm{~F}$-florbetaben, while ${ }^{18} \mathrm{~F}$-florbetapir is a stilbene derivative with a very similar structure.

${ }^{11} \mathrm{C}$-PiB as well as ${ }^{18} \mathrm{~F}$-florbetapir have been used as tracers for cardiac amyloid in patients with ATTR and AL cardiac amyloidosis [18, 19]. However, ${ }^{11} \mathrm{C}-\mathrm{PiB}$ is only available in centers with an on-site cyclotron. Manwani et al. evaluated cardiac uptake with ${ }^{18} \mathrm{~F}$-florbetapir PET in patients with systemic AL amyloidosis and cardiac involvement before and after treatment, as well as its serial utility in monitoring in 15 patients [20]. There was a suggestion that treatment-naïve patients may have higher cardiac uptake. In addition, correlation of myocardial ${ }^{18} \mathrm{~F}$-florbetapir uptake with histological findings in 20 amyloidosis patients (10 AL and 10 ATTR) versus 10 control subjects revealed significantly lower specific ${ }^{18}$ F-florbetapir binding in controls $(p=0.002)$ [21]. Specific ${ }^{18} \mathrm{~F}$-florbetapir binding in AL samples was significantly higher than in ATTR samples $(p=0.001)$. Furthermore, increase in ${ }^{18} \mathrm{~F}$-florbetapir binding on autoradiography correlated well with increasing echocardiography-derived LV wall mass, due to more advanced stages of the disease. More importantly, ${ }^{18} \mathrm{~F}$-florbetapir binding was already present in small amounts before LV wall thickness increased.

A recent systematic review of the application of PET imaging with ${ }^{11} \mathrm{C}$-PiB, ${ }^{18} \mathrm{~F}$-florbetapir and ${ }^{18} \mathrm{~F}$-florbetaben in six studies ( $n=98$ subjects) demonstrated a sensitivity of $92 \%$ and a specificity of $83 \%$ for the detection of AL and ATTR CA [22]. Further, regular bone scintigraphy has emerged as a reliable, non-invasive tool to diagnose cardiac amyloidosis due to ATTR (either ATTRv or ATTRwt), with a sensitivity of $92.2 \%$ (95\% CI 89-95\%) and a specificity of $95.4 \%$ [23, 24]. The use of iodine-123 labelled metaiodobenzylguanidine ( $\left.{ }^{123} \mathrm{I}-\mathrm{MIBG}\right)$, a chemical modified analogue of norepinephrine, is well established in patients with heart failure and also plays an important role in the evaluation of sympathetic innervation in cardiac amyloidosis [25].

Current treatments are targeted at reducing the production of or stabilisation of the precursor protein of amyloid deposits and thereby aim to stop or slow down further accumulation of amyloid. In AL amyloidosis, treatment is directed against light chain-producing plasma cells in order to normalize the light chain serum levels. Recently, genesilencing oligonucleotide drugs that inhibit hepatic synthesis of the precursor protein transthyretin proved to be 
effective in hereditary ATTR amyloidosis [26, 27]. Progression of peripheral polyneuropathy almost ceases entirely and progression of cardiac manifestations may also be halted, or even reversed [28]. Another treatment approach for ATTR CA is stabilization of the TTR tetramer, thereby interfering with the supply of precursor protein and resulting in slowing down of disease progression [29]. Molecular imaging should be able to visualize regression of CA under these new treatment regimens, but data are lacking at this moment. However, these new drugs probably do not inhibit synthesis of variant transthyretin by the choroid plexus in the central nervous system (CNS) leading to meningeal-vascular ATTRv amyloid deposition in the long-term, i.e. $10-15$ years. This may become a new challenge in ATTRv patients, as these drugs are expected to improve life expectancy far beyond the current survival without treatment. Future studies with molecular imaging are necessary to assess the extent of the CNS manifestations. ${ }^{11} \mathrm{C}$-Pib-PET scan currently appears to be the best imaging technique for early detection of these CNS manifestations, by showing - in a presymptomatic state - a pattern different from that seen in Alzheimer's disease [30]. Imaging with ${ }^{18} \mathrm{~F}$-florbetaben-PET/CT is also promising as it may visualize both intra-cerebral deposition of amyloid as well as deposition in other organs, particularly the heart.

Several treatments aiming at promoting amyloid removal are currently being investigated. These treatments, based on the use of monoclonal antibodies and small compounds, employ immunological mechanisms to clear amyloid deposits. Understanding the biochemical structure of amyloid fibrils and the composition of amyloid deposits has been and is of great importance in the development of these drugs. Proteomics may teach us about the specific composition of amyloid and surrounding tissue in order to develop new tracers that specifically target cardiac amyloid [31, 32]. Molecular imaging might be used in the development of new drugs to confirm the binding of compounds or antibodies to the amyloid in vivo (see Fig. 1). As already stated, molecular imaging is also going to play an important role by visualizing the effects of these treatments on the amyloid load.

In summary, a bright future lies ahead for molecular imaging in defining CA, the extent of systemic amyloid manifestations and in treatment monitoring. For diagnostic considerations, specific target imaging using hybrid or multimodality techniques such as PET/MR definitely will play a role in the future. Simultaneous combination of myocardial functional patterns, tissue characterization and visualizing specific targets in CA will yield a potential advantage of PET/MRI application. Several aspects have to be elucidated yet, but as therapeutic possibilities in amyloidosis finally break through, molecular imaging might be a perfect tool to guide the clinician in treating the patient.

\section{Compliance with ethical standards}

Conflict of interest Riemer H.J.A. Slart declares that he has no conflict of interest. Andor W.J.M. Glaudemans declares that he has no conflict of interest. Walter Noordzij declares that he has no conflict of interest. Johan Bijzet declares that he has no conflict of interest. Bouke P.C. Hazenberg received some consultancy fees from Pfizer and Alnylam. Hans L. A. Nienhuis received some consultancy fees from Pfizer and Alnylam.

Ethical approval This article does not contain any studies with human participants performed by any of the authors.

\section{References}

1. Maurer MS, Elliott P, Comenzo R, Semigran M, Rapezzi C. Addressing common questions encountered in the diagnosis and management of cardiac amyloidosis. Circulation. 2017;135:135777.

2. Gonzalez-Lopez E, Gallego-Delgado M, Guzzo-Merello G, de Haro-Del Moral FJ, Cobo-Marcos M, Robles C, et al. Wild-type transthyretin amyloidosis as a cause of heart failure with preserved ejection fraction. Eur Heart J. 2015;36:2585-94.

3. Benson MD, Buxbaum JN, Eisenberg DS, Merlini G, Saraiva MJM, Sekijima Y, et al. Amyloid nomenclature 2018: recommendations by the International Society of Amyloidosis (ISA) nomenclature committee. Amyloid. 2018;25:215-9.

4. Lousada I, Comenzo RL, Landau H, Guthrie S, Merlini G. Light chain amyloidosis: patient experience survey from the amyloidosis. Research consortium. Adv Ther. 2015;32:920-8.

5. Mohammed SF, Mirzoyev SA, Edwards WD, Dogan A, Grogan DR, Dunlay SM, et al. Left ventricular amyloid deposition in patients with heart failure and preserved ejection fraction. JACC Heart Fail. 2014;2:113-22.

6. Martinez-Naharro A, Treibel TA, Abdel-Gadir A, Bulluck H, Zumbo G, Knight DS, et al. Magnetic resonance in transthyretin cardiac amyloidosis. J Am Coll Cardiol. 2017;70:466-77.

7. Hazenberg BP, van Rijswijk MH, Piers DA, Lub-de Hooge MN, Vellenga E, Haagsma EB, et al. Diagnostic performance of 123Ilabeled serum amyloid $\mathrm{P}$ component scintigraphy in patients with amyloidosis. Am J Med. 2006;119:355.e15-24.

8. Biancalana M, Koide S. Molecular mechanism of Thioflavin-T binding to amyloid fibrils. Biochim Biophys Acta. 2010;1804: 1405-12.

9. Stats MA, Stone JR. Varying levels of small microcalcifications and macrophages in ATTR and AL cardiac amyloidosis: implications for utilizing nuclear medicine studies to subtype amyloidosis. Cardiovasc Pathol. 2016;25:413-7.

10. Ng QKT, Sethi P, Saunders TA, Pampaloni MH, Flavell RR. Discordant findings on $18 \mathrm{~F}-\mathrm{NaF}$ and $99 \mathrm{mTc}-\mathrm{HDP}$ bone scans in a patient with ATTR cardiac amyloidosis. Clin Nucl Med. 2018;43: e89-92.

11. Susuki S, Ando Y, Sato T, Nishiyama M, Miyata M, Suico MA, et al. Multi-elemental analysis of serum and amyloid fibrils in familial amyloid polyneuropathy patients. Amyloid. 2008;15:10816.

12. Morton KA. Extra-skeletal uptake of bone agents. J Nucl Med Technol. 1999;27:51-3.

13. Nakanishi T, Yoshioka M, Moriuchi K, Yamamoto D, Tsuji M, Takubo T. S-sulfonation of transthyretin is an important trigger step in the formation of transthyretin-related amyloid fibril. Biochim Biophys Acta. 2010;1804:1449-56. 
14. Aprile C, Marinone G, Saponaro R, Bonino C, Merlini G. Cardiac and pleuropulmonary AL amyloid imaging with technetium-99m labelled aprotinin. Eur J Nucl Med. 1995;22:1393-401.

15. Cardoso I, Pereira PJ, Damas AM, Saraiva MJ. Aprotinin binding to amyloid fibrils. Eur J Biochem. 2000;267:2307-11.

16. Jonker DL, Hazenberg BP, Nienhuis HL, Slart RH, Glaudemans AW, Noordzij W. Imaging cardiac innervation in hereditary transthyretin (ATTRm) amyloidosis: a marker for neuropathy or cardiomyopathy in case of heart failure? J Nucl Cardiol. 2018. https://doi.org/10.1007/s12350-018-01477-y

17. Kircher M, Ihne S, Brumberg J, Morbach C, Knop S, Kortüm KM, et al. Detection of cardiac amyloidosis with 18F-Florbetaben-PET/ $\mathrm{CT}$ in comparison to echocardiography, cardiac MRI and DPDscintigraphy. Eur J Nucl Med Mol Imag. 2019. https://doi.org/10. 1007/s00259-019-04290-y

18. Antoni G, Lubberink M, Estrada S, Axelsson J, Carlson K, Lindsjo $\mathrm{L}$, et al. In vivo visualization of amyloid deposits in the heart with 11C-PIB and PET. J Nucl Med. 2013;54:213-20.

19. Dorbala S, Vangala D, Semer J, Strader C, Bruyere JR Jr, Di Carli $\mathrm{MF}$, et al. Imaging cardiac amyloidosis: a pilot study using 18Fflorbetapir positron emission tomography. Eur J Nucl Med Mol Imaging. 2014;41:1652-62.

20. Manwani R, Page J, Lane T, Burniston M, Skillen A, Lachmann HJ, et al. A pilot study demonstrating cardiac uptake with 18Fflorbetapir PET in AL amyloidosis patients with cardiac involvement. Amyloid. 2018;25:247-52.

21. Park MA, Padera RF, Belanger A, Dubey S, Hwang DH, Veeranna $\mathrm{V}$, et al. 18F-Florbetapir binds specifically to myocardial light chain and transthyretin amyloid deposits: autoradiography study. Circ Cardiovasc Imaging. 2015;8(8). https://doi.org/10.1161/ CIRCIMAGING.114.002954

22. Kim YJ, Ha S, Kim YI. Cardiac amyloidosis imaging with amyloid positron emission tomography: a systematic review and meta-analysis. J Nucl Cardiol. 2018. https://doi.org/10.1007/s12350-018$1365-\mathrm{x}$

23. Glaudemans AW, van Rheenen RW, van den Berg MP, Noordzij W, Koole M, Blokzijl H, et al. Bone scintigraphy with (99m)techne- tium-hydroxymethylene diphosphonate allows early diagnosis of cardiac involvement in patients with transthyretin-derived systemic amyloidosis. Amyloid. 2014;21:35-44.

24. Treglia G, Glaudemans AWJM, Bertagna F, Hazenberg BPC, Erba PA, Giubbini R, et al. Eur J Nucl Med Mol Imaging. 2018;45(11): 1945-55.

25. Slart RHJA, Glaudemans AWJM, Hazenberg BPC, Noordzij W. Imaging cardiac innervation in amyloidosis. J Nucl Cardiol. 2019;26(1):174-87.

26. Adams D, Gonzalez-Duarte A, O'Riordan WD, Yang CC, Ueda M, Kristen AV, et al. Patisiran, an RNAi therapeutic for hereditary transthyretin amyloidosis. N Engl J Med. 2018;379:11-21.

27. Benson MD, Waddington-Cruz M, Berk JL, Polydefkis M, Dyck PJ, Wang AK, et al. Inotersen treatment for patients with hereditary transthyretin amyloidosis. N Engl J Med. 2018;379:22-31.

28. Solomon SD, Adams D, Kristen A, Grogan M, González-Duarte A, Maurer MS, et al. Effects of Patisiran, an RNA interference therapeutic, on cardiac parameters in patients with hereditary transthyretin-mediated amyloidosis. Circulation. 2019;139:431-43.

29. Maurer MS, Schwartz JH, Gundapaneni B, Elliott PM, Merlini G, Waddington-Cruz $\mathrm{M}$, et al. Tafamidis treatment for patients with transthyretin amyloid cardiomyopathy. N Engl J Med. 2018;379: 1007-16.

30. Sekijima Y, Yazaki M, Oguchi K, Ezawa N, Yoshinaga T, Yamada $\mathrm{M}$, et al. Cerebral amyloid angiopathy in posttransplant patients with hereditary ATTR amyloidosis. Neurology. 2016;87:773-81.

31. Brambilla F, Lavatelli F, Di Silvestre D, Valentini V, Palladini G, Merlini G, et al. Shotgun protein profile of human adipose tissue and its changes in relation to systemic amyloidoses. J Proteome Res. 2013;12:5642-55.

32. Di Silvestre D, Brambilla F, Mauri PL. Multidimensional protein identification technology for direct-tissue proteomics of heart. Methods Mol Biol. 2013;1005:25-38.

Publisher's note Springer Nature remains neutral with regard to jurisdictional claims in published maps and institutional affiliations. 